Meta

Journal des traducteurs

Translators' Journal

\title{
Comment peut-on traduire Hafiz... ou Freud ?
}

\section{Bernard This et Pierre Thèves}

Volume 27, numéro 1, mars 1982

Psychanalyse et traduction

URI : https://id.erudit.org/iderudit/004477ar

DOI : https://doi.org/10.7202/004477ar

Aller au sommaire du numéro

Éditeur(s)

Les Presses de l'Université de Montréal

ISSN

0026-0452 (imprimé)

1492-1421 (numérique)

Découvrir la revue

Citer cet article

This, B. \& Thèves, P. (1982). Comment peut-on traduire Hafiz... ou Freud ? Meta 27(1), 37-59. https://doi.org/10.7202/004477ar d'utilisation que vous pouvez consulter en ligne.

https://apropos.erudit.org/fr/usagers/politique-dutilisation/ 


\title{
COMMENT PEUT-ON TRADUIRE HAFIZ... OU FREUD?
}

\author{
Bernard This et Pierre Thèves
}

Il en est peut-être du poète comme d'un parfum ou d'une saveur: sans vouloir ni pouvoir en distinguer les essences, certains les reconnaissent et parffois s'en enivrent. Les poètes persans depuis toujours m'enchantent, et je ne parle pas le persan! La poésie et son transfert de langue à langue toucheraientils un sixième sens, comme par intuition, où l'oreille, le parler, le chant et la musique susciteraient une connivence singulière entre artisans du même métier, alchimiste du même amour? Ou tout cela ne serait-il qu'ambition démesurée et présomption?

A travers les nombreuses lectures de Hafiz qui me furent faites dans sa langue, et les traductions simultanées, mot à mot, j'ai rencontré, connu et suivi, "celui qui savait le Coran par cœur». Ensuite, grâce aux traductions savantes auxquelles je dois tout - merci MM. Defremery, Nicolas, Arthur Guy, Henri Massé, Roger Lescot, Gilbert Lazard, Vincent Monteil - j'ai pu approcher au plus près du poète. Les Ghâzels du "rossignol de Chiraz", écrits et chantés à la gloire du vin et de l'amour, leurs reflets et leurs mouvements, leurs audaces et quelques-uns de leurs secrets me sont devenus familiers. Émerveillé de voir un objet de pensée et de langage s'ouvrir comme une fleur à la pointe du calame, j'ai longtemps, très longtemps, écouté, respiré, ausculté Hafiz, vers par vers. Grâce à eux, sans lesquels rien n'aurait pu être entrepris, prédécesseurs et amis de «l'alchimie du Verbe», je tente à mon tour d'offrir aujourd'hui une transposition jumelle qui soit «aussi près du poète que l'est sa veine jugulaire" comme il est écrit dans le Coran.

Dans une aventure faite de respect et de repentir, je ne souhaite que servir un Maître. Bien conscient de mes insuffisances, mais imbibé du vin de poésie, me sera-t-il parfois arrivé de parler Hafiz? Si oui, que Dieu - et des années de travail - en soient loués! Faudrait-il aller plus avant? Toute traduction se pétrit lentement et se reprend sans cesse, sans fin remodelée dans l'écriture comme dans ses échos. C'est le plus souvent dans l'inconscience que l'architecte du langage, le prospecteur de l'homologue édifie ce qu'il réinvente. Pour Hafiz, j'ai tenté de ressusciter une «syntaxe respiratoire», de retrouver la gorge et la mémoire. Certain que la poésie ne se retrouve pas dans la copie de la marquetterie du vocable, mais plutôt dans le chant - chant profond «canto jondo», profération et murmure - j'ai choisi d'accorder ma voix à celle du poète, ma démarche et mon pas aux siens. Une fois le sens respecté, les images reprises, j'ai délibérément tourné le dos au formalisme, c'est-à-dire au décalque, pour 
mieux servir la vie. Chaque langue invente ses déambulatoires, ses miroirs sans tain, ses passerelles.

Pour être fidèle à la terminologie de Valery Larbaud, grand maître de la traduction des poètes, j'ai qualifié mon travail (amour et enfantement) de «version . L'adaptation supposait une traduction trop libre, trop proche d'un arrangement qui sacrifie au goût du jour. L'interprétation ouvrait la porte à l'arbitraire, la paraphrase se perdait dans ses sinuosités ornementales. Toute sécheresse devient leçon d'anatomie, tout excès d'élégance relègue l'essentiel au second rang. Il en est du langage des poètes comme des trésors : il faudrait une oreille ultra-sensible pour enregistrer les déclics, des doigts aimantés pour forcer, sans forcer, des serrures! Curieux et passionnant travail, «un grand art modeste" disait Elsa Triolet, où chaque mot, chaque virgule remettent l'édifice en question. Ma version des vers de Hafiz (en persan, un «vers» est, en fait, un distique) est donc une version libre."

C'est ainsi que Pierre Seghers ${ }^{1}$, dans «le livre d'or du Divân» aborde le problème de la traduction en poésie.

J'en étais là, dans ma lecture du «divân», quand j'ai reçu un petit mot de François Peraldi me demandant de participer à un numéro consacré à une «réflexion sur psychanalyse et traduction»; j'ai pensé qu'il conviendrait de comparer les difficultés de la traduction d'un texte poétique - Hafiz par exemple - et d'un texte plus rigoureux, celui de Freud en l'occurrence, mais aussitôt le titre de cet article s'est formulé : «Comment peut-on traduire Hafiz... ou Freud?», laissant entendre, par cette interrogation, qu'il s'agit d'une tâche impossible.

Comment traduire un poème? Je l'ignore, et la lecture du livre de Seghers ne me permet pas d'en dire plus. Pour Freud, je devrais le savoir, puisqu'avec Pierre Thèves nous avions fait paraître, en 1975, la traduction d'un article, relativement court mais essentiel: Die Verneinung. C'est même ce travail qui a donné à François Peraldi l'idée de s'adresser à nous.

Blanchot ayant écrit : "la responsabilité dont je me suis chargée n'est pas la mienne et fait que je ne suis plus moi», pouvons-nous soutenir que la responsabilité de cette traduction n'est pas la nôtre? Elle nous engage certes nominalement, mais n'engage-t-elle que nous deux? Sûrement pas, puisqu'ayant procédé, en des temps successifs, à l'étude des «versions» disponibles en langue française, c'est à partir du travail de ces divers traducteurs que notre réflexion s'est développée: c'est à partir de leurs traductions que notre article s'est élaboré. "Nos opinions, disait Montaigne, s'entent les unes sur les autres. La première sert de tige à la seconde. Nous eschellons ainsi de degrés en degrés. Et advient de là que le plus haut monté a souvent plus d'honneur que de mérite, car il n'est monté que d'un grain sur les épaules du pénultieme». Qu'importe le mérite! Escheller n'est pas forcément exceller. Pour libérer l'auteur de l'enflure où il se complairait volontiers, rien de mieux qu'un travail de groupe, ou qu'un groupe au travail ! Vient toujours un moment où la traduction proposée

1. Pierre Seghers (1978): op. cit., p. 163. 
est améliorée par d'autres : notre travail de 1975 que François Peraldi qualifiait de «modèle du genre» fut «repris» vertement par certains amis qui se chargèrent d'ouvrir nos yeux sur les erreurs que nous avions commises. Notre "traduction 1981 », dix-septième version, est loin d'être parfaite, mais c'est la dernière en date, disponible au Coq Héron.

Le comité de rédaction de cette revue nous ayant menacés de rééditer le $n^{\circ} 52$ (de 1975), qui était demandé par de nombreux correspondants, nous avons préféré laisser paraître cette nouvelle traduction, mais nous savons que certains points restent litigieux. Nous nous en expliquerons ici. Nous savons maintenant, pour l'avoir expérimenté et en avoir souffert, qu'aucune traduction n'est parfaite et définitive. Nous reprendrons ce texte dans les années à venir, nous serons «travaillés» par lui, dans la mesure où (tripalium) nous accepterons encore de le travailler. Nous ne savons pas où nous mène ce texte, mais nous avons réalisé (Pathein Mathein - tout savoir impliquant souffrance) qu'il nous avait modifiés. «Cette responsabilité dont je me suis chargé... fait que je ne suis plus moi » écrivait Blanchot. Ce travail nous a-t-il transformés, métamorphosés, fait autres? Si le grec dit morphé, le latin dit forma, vous voyez la curieuse inversion des consonnes! Il faut noter que trans - à travers - n'est pas méta - à côté; la traductio n'est pas la métaphasis; cependant ce sont les mots que les latins et les grecs utilisent pour désigner cette opération. Traduire, en français, c'est conduire (ducere) à travers (trans), mener le troupeau des mots en traversant la frontière. En allemand, traduire c'est übersetzen, mettreplacer au dessus : le mot recouvre, surmonte, surpasse, domine, prédomine, et le «passage» ressemble à une opération guerrière : il y a dans cette élévation du mot une menace, voire une violence meurtrière, une surcharge (überschreiben) qui écrase et fait disparaître ce qui était primitivement inscrit (Übertragen c'est transcrire). Si l'écrit se dit Schrift, l'Überschrift, traduit par transcription, est une surcharge, un écrit sur un autre. Que devient le premier écrit? Il est supprimé, réprimé, passé dans les dessous, sous silence (unterdrücken).

Traduttore - traditore nous rappelait Freud, dans «le mot d'esprit et ses rapports avec l'inconscient". Traduire - trahir! "La similitude des deux mots, frisant l'identité, exprime de façon saisissante la fatalité qui fait du traducteur un traître à son auteur». Est-ce seulement pour nous déculpabiliser que nous prenons notre plume, désirant faire réapparaître, dans le commentaire, ce qui a disparu dans la traduction? L'interprète n'est pas le traducteur: vous voyez tout de suite la différence; il est entre les deux, il va et vient de l'un à l'autre, prêtant à chacun ce qui lui manque pour comprendre le message. L'interprète doit se faire «anagnoste», c'est-à-dire «lecteur», dans la mesure où la «lecture» d'un texte, anagnosis, fait revenir «en arrière», en ce temps de l'écriture, où celui qui l'inscrivait pouvait encore en répondre, et le commenter. Le lecteur du texte peut le commenter.

La tradition médiévale voulait que le commentaire portât sur trois points :

- la lettre, c'est-à-dire l'explication grammaticale,

- le sens, l'intelligence du texte,

- la sentance, son sens profond, le contenu doctrinal. 
De nombreux manuscrits présentent, au centre de la page, le texte étudié avec, dans les marges, les diverses gloses se rapportant à la littera, au sensus, à la sententia. Pour des raisons typographiques, nous n'avons pas pu adopter cette disposition, dans notre traduction, mais nous avons placé le texte français en face du texte allemand, nos commentaires ne cessant de soutenir cet affrontement.

L'écriture qui, toujours, comme la traduction, possède un petit côté parricide, faux-frère, traitre, infidèle (c'est sa dimension thanatophore) nous permet cependant de remédier à la violence de cette «mise à mort» qu'est tout Übersetzung qui veut imposer l'hégémonie d'un seul sens, l'unique, inique. Elle permet la comparaison des diverses versions; nous avons numéroté les versions disponibles pour les faire «jouer» ensemble. Un professeur, après les avoir «corrigées », les classerait par ordre de mérite; nous avons préféré les ordonner en fonction de leur date d'apparition, les présentant comme autant d'interprétations musicales, leurs différences donnant au texte freudien un relief qui ne cessera de se creuser et de nous interroger. Car, c'est bien là le scandale de la traduction, aucune phrase n'est traduite de semblable façon par l'ensemble des premiers traducteurs.

« Cette responsabilité dont je me suis chargé... fait que je ne suis plus moi», dans la mesure où mon oreille perçoit mieux les dissonnances; la polyphonie provoque notre réflexion, débusque notre paresse, nos refus, notre inhospitalité intellectuelle, nos réticences à nous laisser travailler par ce qui insiste cependant dans le message: nous nous hâtons, trop souvent, à donner ou plaquer du sens, au lieu de nous laisser traverser par les diverses possibilités de la structure combinatoire. Il n'existe pas de lecture unique, objective, innocente: si toute lecture est déjà une interprétation, le lecteur donnant sens aussitôt, que dire de la traduction? Mais ayant entrepris ensemble ce travail, nous devions tous deux ajuster et confronter nos interprétations. Faisant état de notre travail dans divers cartels, ou groupes de travail, nous en recevions des critiques, rapidement. C'est pourquoi nous pouvons soutenir que «la responsabilité» de ce texte n'est pas seulement «la nôtre»: nous avons sans cesse cheminé, dans le parcours de cette lecture, avec des compagnons réels ou imaginaires qui nous précédaient ou nous suivaient.

S'il arrive, chemin faisant, que le traducteur porte, un instant, sur ses épaules, un texte léger, insignifiant, il n'en est comme "passeur» que bien peu affecté; ce texte est alors rapidement déposé, oublié. Mais, saint Christophe ne pourra pas le nier, il arrive aux passeurs de singulières aventures : cet enfant dont il avait chargé ses épaules, n'était-il pas, au milieu de la rive, devenu si lourd qu'il avait fallu que le passeur s'arqueboute, de toutes ses forces, sur son bâton? Et, me croirez-vous, enfoncé dans le lit du fleuve, ce bâton s'était transformé en un arbre puissant et solitaire, enraciné au milieu du courant.

Christophe portait le Christ, le Christ portait le monde. Miracle, intrusion du symbolique dans le réel! Me direz-vous sur quoi s'appuyait le passeur quand il se fit «Porteur de la Parole»?

Et Freud, quand il fit parvenir à son ami W. Fliess cette «lettre $52 »$, du 6 décembre 1856, entre 1'Esquisse et la Traumdeutung, sur quoi prend-il appui, 
si ce n'est sur ses rêves et ceux de ses patients, dont il découvre qu'ils sont à lire, comme une écriture, avant d'en faire un livre.

Je veux mettre en relief que les inscriptions qui se suivent l'une l'autre représentent l'accomplissement psychique d'époques successives de la vie. $C^{\prime}$ 'est à la frontière de deux de ces époques que la traduction du matériel doit s'effectuer. La particularité des psychonévroses, je me les explique en ceci que la traduction, pour certains matériaux, ne s'est pas effectuée, ce qui a certaines conséquences... La défaillance (Versagung) de la traduction, c'est ce qui s'appelle cliniquement le refoulement (Verdrängung). Le motif de celui-ci est toujours une déliaison (Entbindung) de déplaisir qui se produirait par traduction, comme si ce déplaisir provoquait une perturbation de la pensée qui n'admettrait pas le travail de traduction.

Prenant quelques exemples, Freud écrit : «L'accès de vertige, la crise de larmes, tout est réglé (berechnet - calculé) sur l'autre, mais dans la plupart des cas pour cet autre préhistorique, inoubliable, que personne ensuite n'atteindra plus... Un de mes patients gémit encore maintenant dans son sommeil comme autrefois (pour que sa maman, qui est morte quand il avait 22 mois, le prenne avec elle)». Ce symptôme que Freud appelle Bettsucht - passion du lit - est un appel. Dans la «nuit obscure» du travail de traduction, il arrive que la pensée refuse le travail de traduction, c'est le refoulement: nos analyses personnelles s'avéraient nouées aux passages problématiques. Nous aurions voulu demander à Freud ce qu'il avait l'intention d'exprimer, nous aurions voulu faire appel à lui, mais devant l'écrit nous sommes comme des orphelins, sans parents, et l'autre préhistorique ne vient pas nous prendre dans ses bras!

«Travaillez, prenez de la peine, c'est le fond qui manque le moins». Et Boileau fait écho au fabuliste :

Vingt fois sur le métier remettez votre ouvrage,

Polissez-le sans cesse et le repolissez.

Ajoutez quelquefois et souvent effacez.

Mais, voilà, quand nous avons traduit un texte, quand nous nous en sommes séparés, il n'est plus possible de faire marche arrière : ce qui est écrit nous engage. Notre réflexion se poursuivant, nous ne pouvons plus reprendre le texte, c'est pourquoi, par des commentaires expliquant nos choix et nos décisions, nous tentons de récupérer ce que la traduction nous a fait perdre inévitablement.

Il faut noter que la défaillance ou le défaut de traduction dont parlait Freud, dans sa lettre 52, ne se présente pas comme une simple erreur; c'est un lapsus, significatif, une action où le «dire» (sagen) échoue, rate, fait défaut, pour des raisons précises qu'il convient d'élucider. Ce n'est pas das Versagen, la défaillance par non fonctionnement ou panne, c'est die Versagung l'action de dire à côté, en plus... au-delà.

C'est toute la fonction de cette particule allemande ${ }^{2}$ (que nous retrouverons dans Die Verneinung) qu'il faudrait expliquer au lecteur; cette particule indique que l'action est menée jusqu'au bout, mais, sous son influence, le verbe

2. Que Freud aimait tout particulièrement. 
se modifie de bien curieuse façon. Ce préfixe de composition "Ver» (prononcer "fer") $)^{3}$ exprime :

- la transformation : klein - petit, verkleinern - rendre petit. gross - grand : vergrössern, rendre grand. süss - doux : versüssen, adoucir.

- le changement d'état : Stein - la pierre; versteinen - pétrifier. golden - en or; vergelden, dorer.

- l'intensification de l'action:

lassen: laisser; verlassen, quitter irren : errer; sich verirren, se perdre trinken : boire; vertrinken, se noyer en buvant spielen : jouer; verspielen, dépenser en jouant schwinden : décroître; verschwinden, disparaître decken: couvrir; verdecken, cacher graben: creuser; vergraben, enterrer bluten: saigner; verbluten, perdre tout son sang hungern : avoir faim; verhungern, mourir de faim urteilen : juger; verurteilen, condamner.

- la suite extrême d'une action:

klingen : sonner; verklingen, cesser de sonner scheiden: séparer; verscheiden, mourir

- son contraire, qui n'est qu'une forme d'extrêmisation: achten : estimer; verachten, mépriser bieten : offrir; verbieten, interdire kaufen : acheter; verkaufen, vendre wünschen: désirer, souhaiter; werwünschern, exécrer, maudire, ensorceler, enchanter.

Un cycle se termine et, insensiblement, un autre commence : la particule «Ver», à ce moment solsticial, fait basculer le sens en son contraire.

Mais revenons à cet article que nous avons traduit : Die Verneinung. Nein c'est «non». Neinen n'existant pas, ni beneinen, pour nier l'allemand utilise le préfixe de composition «Ver»: verneinen c'est répondre négativement. Et il n'y a pas d'hésitation avec cette particule, c'est «non» absolument. La Verneinung sera d'abord l'action de "dire-non", la négation (apophasis en grec) mais ce terme se meut dans cet espace où tout peut se modifier et se renverser. Et nous passons de la "négation" à la «dénégation» insensiblement. Alors, comment traduire ce titre?

H. Hoesli le traduisit en 1934 pour la Revue française de psychanalyse ${ }^{4}$, sous le titre de «la négation».

J. Lacan ${ }^{5}$, dans une communication faite à la Société anglaise de psychanalyse, le 2 mai 1951, avait souligné l'importance de la Verneinung: «... nous avons appris à être sûrs que, lorsque quelqu'un dit: "Ce n'est pas ainsi», c'est parce que «c'est ainsi», et quand il dit: «Ce n'est pas cela que je veux

3. Mais, oui, c'est ici et maintenant qu'il faut commencer à parler l'allemand, avec nous. Vous verrez, pour lire Freud, il n'y a pas d'autre solution! Apprenez d'urgence l'allemand. C'est passionnant, comme un... roman policier.

4. Tome VII, $\mathrm{n}^{\circ} 2$, p. 174-177.

5. «Quelques réflexions sur l'Égo», publié dans le Journal internat. de Psychanalyse. 1953, vol. 34, p. 11-17. 
dire» il dit vraiment». Citant l'article de Freud, Lacan y voyait la justification de «l'épigramme du philosophe disant que le langage fut donné à l'homme pour cacher ses pensées. Notre vue, c'est que la fonction essentielle de l'Ego est très proche d'une méconnaissance systématique de la réalité à laquelle les analystes français se réfèrent en parlant de psychose».

En 1954, Lacan, dans son séminaire du 10 février, proposa à Jean Hyppolite de commenter la Verneinung: "Il vaudrait mieux la traduire par la dénégation " précisait-il. "Verneinung, comme M. Hyppolite me le faisait remarquer tout à l'heure, c'est dénégation et non pas négation comme on l'a traduit en français. C'est toujours ainsi que je l'ai moi-même évoquée dans mon séminaire, chaque fois que j'en ai eu l'occasion».

«Négation» ou "Dénégation»? D'emblée le problème est posé. Le titre, immédiatement, exige que nous prenions parti, pour ou contre.

Opter pour «la négation», c'est choisir la traduction de la «Société parisienne de psychanalyse», reconnue officiellement par la «Société internationale de psychanalyse». Un «comité de traduction» impose la version officielle que l'on doit citer ${ }^{6}$. Risquer «la dénégation » c'est se ranger du côté de Lacan, et d'Hyppolite, en contestant «la psychologie de l'Ego» et en refusant d'obéir au «comité de traduction». Vérité ou confort au sein d'un groupe qui vante le «Moi» fort, autonome, maître de la réalité? Avec la Verneinung nous sommes au cœur du conflit qui divise les analystes : l'EGO, fonction de méconnaissance, ou de maîtrise et de conscience.

Alors que le procès de la dénégation, marquée déjà dans la grammaire par l'existence du «ne» explétif, est toujours le fait d'un acte de la parole, la traduction «dénégation»s'élève à la propriété de cet acte qui pose un «je parle» en face d'un «je suis parlé » - «Je parle» et je suis le maître de la parole, créant les mots, ou «parle-être», je suis fondamentalement marqué, déterminé par la parole qui pré-existe à mon existence de sujet? Le maintien, en français, du préfixe, et sa transformation française en "dé", résultent-ils d'une lecture que le texte lui-même justifie?

La question clinique qui clôt la première partie de l'article, à la fin du troisième paragraphe ( nous réussissons à vaincre la dénégation, elle aussi, et à obtenir la pleine acceptation intellectuelle du refoulé - le processus même du refoulement, par là, n'est pas encore levé»), donne le pas à l'abord métapsychologique de la question, ce qui renverse la question clinique : la non-levée du refoulement concerne la constitution du refoulement lui-même. L'Ursprung, dont Freud parle en ce début du $4^{\mathrm{e}}$ paragraphe, n'est autre que le saut (la tra-ductio) que représente la «reprise» de la «sorcière métapsychologie». Et J. Lacan écrira, à ce moment de sa lecture, en écho au vif du propos freudien : «peut-on se contenter de parler ici de refoulement?» (Écrits, p. 379). C'est à ce moment, précisément, qu'il invitera Hyppolite à prendre la parole pour convenir avec lui du juste choix du terme français «dénégation». On sait vers

6. C'est ainsi qu'il me fut imposé d'écrire «le moi doit déloger le ça» pour «Wo es war soll ich werden» (B.T. Revue franc. de Psycha.). 
quels «hauts cols», Lacan placera le débat métapsychologique (Écrits, p. 282 283). C'est ce parcours que les analystes alourdis par le souci de promouvoir le «moi fort» et conscient, produit par l'analyse, ne pourront effectuer: après l'analyse la libido est devenue «parfaitement objectale», l'analysé purifié, transformé, devenu «conscient» de ses difficultés, est un «sage». Merveilleuse publicité ? Illusion naïve?

A la reprise, par Freud, des premiers éléments de son «Esquisse», que l'élaboration de la seconde topique réoriente, répond l'articulation, le nouage des catégories du réel, du symbolique, de l'imaginaire, qui fondent un enseignement : affirmation primordiale et jugement d'existence freudiens sont placés au niveau d'un axe de coupure entre réel et symbolique.

La question cruciale de la «Chute du complexe d'Oedipe» est entendue : le procès qui décrit cette chute «est plus qu'un refoulement, il équivaut à la levée du complexe». Dire que le complexe d'Oedipe échoue sous la menace de castration, n'achève pas le problème, «il reste l'espace pour une spéculation théorique qui renverse le résultat acquis, ou qui est capable de le mettre sous un nouveau jour». Le texte de la Verneinung réalise ce renversement, dans le passage qu'il effectue d'un discours clinique vers sa «traduction métapsychologique».

«Ver» et «dé», ces préfixes rendent donc parfaitement compte du passage que ce texte franchit. La grammaire est appelée à venir marquer l'événement : événement d'une lecture (1954); la traduction en est solidaire. Si grammaire et traduction font passer dans la langue la trace de la logique d'une lecture, c'est qu'il y a eu interprétation. Celle-ci a un nom: «retour à Freud», par J. Lacan. Elle a donc sa traduction conceptuelle, sa prise de mémoire (Begriffs-erinnerung - Freud) : dénégation. Un acte de traduction a eu lieu : il n'a pas failli; il n'est pas sans dire la frontière ${ }^{7}$, la cime où se croisent réel et symbolique. Cette traduction inscrit, définitivement, cette notion freudienne dans la logique des rapports de la parole et de l'inconscient. En ce sens, à l'instar du trait d'esprit, elle s'apparente à un événement d'un intérêt général (paraphrase de Freud à propos du Witz).

D'autres concepts de Freud ont connu une «frappe» analogue dans l'enseignement de J. Lacan. Rappelons le commentaire sur Schreber qui, enfin, décide de la traduction de Verwerfung par forclusion, celui de l'Éthique qui, dans l'orientation donnée alors au "principe de réalité " dans son voisinage avec le réel, consacre la «chose», le Ding esquissé par Freud. Ces traductions scandent un trajet théorique, et participent du renouvellement de la logique de son élaboration. Leur opérativité est celle que leur assigne l'appareil psychique luimême (lettre 52). Leur réalité d'inscription est de venir traduire la refonte et l'articulation des concepts suivant le temps logique de la construction du champ théorique et clinique.

Reprenons une fois de plus l'oubli du nom de Signorelli : quand, la première fois, Freud le soumet à l'analyse (1898), il écrit : «la traduction de Signor en Herr (Seigneur) était donc le chemin, par lequel l'histoire que j'avais passée

7. Cf. Lettre 52 de Freud à Fliess. 
en dessous (unterdrückt, voir Séminaire $X I)^{8}$ avait entraîné dans le refoulement le nom que je cherchais». Le franchissement, en termes de signification, d'une langue à une autre (Signor veut dire Herr) devient le recours même qu'offre au silence tenu par Freud sur les choses du sexe et de la mort, le fait de traduire: «à Raguse, je parlais constamment l'italien, c'est-à-dire que je m'étais habitué à traduire dans ma tête l'allemand en italien». Freud n'avait pas parlé, n'avait pas dit - il en reste à la signification, il traduit. Toutefois, ce n'est pas sans bénéfice, puisque la voie qu'emprunte la traduction pulvérisera le nom oublié en autant de résonances substitutives que le discours brisé le permettra, et révélera par là même, après coup, tous les frayages de formation de l'inconscient et de la structure du symptôme, enchaînés par l'oubli. Ici la traduction avait accompagné le refoulement. La signification avait pris figure de censure.

Traduire peut avoir d'autres effets. L' "Homme aux loups", au moment même où «il a su traduire " son rêve de la guêpe jaune, écorne le terme allemand de guêpe. Ce faisant, il prononce, dans sa matérialité de lettre, les initiales de son nom patronymique - (S.P.). La traduction, dans cet exemple tiré de la cure, revêt ses titres de noblesse: elle prend effet de l'acte d'énonciation. Elle s'insinue dans l'acte de parole jusqu'au dire de la menace de castration. Freud notera que «le fait de parler une langue étrangère écrante (decken) l'action symptomatique». On mesure la proximité qu'entretiennent alors, dans la constitution d'un discours, la structure du symptôme et son tenant-lieu, à un moment de suspens du dire, la traduction.

Quelle pourrait être cette suspension particulière de la parole, quand s'effectue le passage d'un niveau du discours à un autre, que réalise, dans la cure, le fait de traduire? A quel tenant-lieu d'inscription psychique se tient le fétiche qu'il faut lire en anglais (a glance at the nose) et non en allemand? Cette résurgence de la langue maternelle anglaise supplée-t-elle à quelque défaut symbolique de traduction?

De même, recourir à l'expression too good to be true pour rendre sensible le sentiment d'étrangeté devant l'Acropole, et ainsi fonder un jugement d'existence, donne bien plus de consistance au tracé logique du trouble de mémoire, que de "pouvoir traduire: je ne suis pas digne d'un tel bonheur, je ne le mérite pas». D'un côté l'emprunt à un trésor de la langue frappé de sonorités étrangères, de l'autre l'aplatissement du désarroi logique, en terme de signification.

Tout aussi notable le recours à la terminologie de la psychiatrie française, quand Freud veut faire état des phénomènes de fausses reconnaissances.

Ici, Freud ne traduit plus. Il nomme, à partir de l'autre langue, les processus qu'il tente d'articuler. La suspension de la parole est radicale: il s'agit précisément de la description des phénomènes qui se situent dans les rapports du langage avec le réel. Cette importation de la langue étrangère est mise ainsi en position de fremde Hilfe (secours autre, v. l'Esquisse) quand se fait pressante l'épreuve logique de l'accès au jugement d'existence. Freud n'équivoque pas, ni

8. J. Lacan, Séminaire $X I$, Seuil. 
ne traduit. Nous nous trouvons là devant une des «mesures essentielles» de la parole, mesure qui fait état d'un nouage spécifique des trois registres (I.R.S.). "Quand ce nouage s'équivoque, dans la langue, il n'y a aucun inconvénient à ce qu'on ne traduise rien » dit Lacan en 1977 (Ornicar, ${ }^{\circ}$ 16), position extrême de l'effet de réel dans la langue.

Ces exemples tirés de l'œuvre de Freud viennent illustrer les confins reculés où $\mathrm{J}$. Lacan énonce son souci de serrer au plus près parole et formation analytique: «ne pas passer trop loin de la parole, dont les mesures différentes sont essentielles à l'effet de formation que je cherche» écrivait-il en 1977 (Instance de la lettre), ce que nous mettons en regard de la difficile «accession d'un sujet à une notion comme la Verwerfung à mesure même du fait qu'il y est plus intéressé. Drame quotidien où se rappelle que cet enseignement qui ouvre à tous sa théorie a pour enjeu pratique la formation du psychanalyste" (Écrits, p. 363). Un retour à Freud ne saurait donc se priver de la tâche de traduction dans le travail toujours actuel d'actualisation logique du discours analytique.

L'autre point de rencontre entre parole et traduction, énoncé par Lacan, résulte de l'opération de transformation qu'il a fait subir à l'algorithme de Saussure. Quand est affirmée l'autonomie du signifiant, afin de consacrer la séparation fondatrice de la lettre et de l'être, en celle de l'autre et de l'Autre (Écrits, p. 528) nous pouvons, alors seulement, lire ce qui a présidé à toute l'opération de retournement effectuée : un acte de parole conjoint de celui d'une tra-duction. "Quand je parle de Heidegger ou plutôt quand je le traduis, je m'efforce à laisser à la parole qu'il profère sa signifiance souveraine» (Écrits, p. 528). La dimension d'effort souligne celui de soutenir l'effet de barre. La résistance déployée donne la mesure du renversement de perspective effectué. Ainsi soutenus, parler et traduire se conjuguent. Ici, traduire ne vient pas marquer, désigner le franchissement logique réalisé, mais, porté par l'effort de la parole, traduire devient le mouvement de suspension lui-même, à l'œuvre, en attente de son affranchissement.

Soutenir l'effet de barre incombe, donc, à l'acte de traduire, dans la cure l'énoncé comme effet d'énonciation, dans un texte l'articulation logique comme effet de coupure où le symptôme clinique est alors élevé à sa fonction d'immersion dans le réel.

Mais revenons à la Verneinung, ce texte que Lacan utilise pour ouvrir son premier séminaire sur «les écrits techniques de Freud»: "Cet écrit manifeste une fois de plus la valeur fondamentale de tous les écrits de Freud. Chaque mot mérite d'être mesuré à son incidence précise, à son accent, à son tour particulier». Chaque mot doit être «inséré dans l'analyse logique la plus rigoureuse. C'est en quoi il se différencie des mêmes termes groupés plus ou moins vaguement par des disciples, pour qui l'appréhension des problèmes a été de seconde main, si l'on peut dire, et jamais pleinement élaborée, d'où résulte cette dégradation de la théorie analytique qui se manifeste sans cesse dans ses hésitations".

Une appréhension de «seconde main» c'est précisément celle qui passe par la lecture de Freud, en français, à partir de l'anglais! On peut admirer le 
travail réalisé par Strachey mais on doit aussi le critiquer. La traduction anglaise était donnée, à l'Institut, comme "parfaitement fidèle», on en disait le plus grand bien, hélas! Il est inadmissible de traduire un texte, à partir d'une traduction: l'anglomanie doit avoir des limites! Pour introduire la Verneinung, Lacan ${ }^{9}$ faisait remarquer que

le privilège donné à la lettre de Freud n'a rien chez nous de superstitieux. C'est là où l'on en prend à son aise avec elle, qu'on y apporte une sorte de sacralisation fort compatible avec son ravalement à un usage de routine. Que tout texte, qu'il se propose comme sacré ou profane, voit sa littéralité croitre en prévalence, de ce qu'il implique proprement d'affrontement à la vérité, c'est ce dont la découverte freudienne montre la raison de structure. Précisément en ce que la vérité qu'elle apporte, celle de l'inconscient, doit à la lettre du langage, à ce que nous appelons le signifiant. Ceci, il nous rend compte incidemment de la qualité d'écrivain de Freud, est surtout décisif à intéresser le psychanalyste, aussi loin qu'il le peut, au langage, comme à ce qu'il détermine dans le sujet ${ }^{10}$.

Si «l'effet de vérité qui se livre dans l'inconscient et le symptôme, exige du savoir une discipline inflexible à suivre son contour, car ce contour va au contraire d'intentions trop commodes à sa sécurité », on comprend que les analystes soient si prêts, à leur insu, à trahir le message freudien: "On capitule à le comprendre, pour ne pas le rencontrer, et son «langage» comme on dit, pour réduire un discours au verbal, vient à fleurir dans les propos de l'On le plus lucifuge». Freud trahi par ceux qui s'empressent de sucer sa pensée dans une indécente intimité «redoublant l'horreur de la forcer par la morose opération de l'obstruer», d'où «rien ne vient plus à être manié de chaque joint délicat que Freud emprunte au plus subtil de la langue, sans que d'avance y soient coulées les images confuses où se précipitent ses plus basses traductions».

Comment oser traduire Freud après une telle mise en garde? En abandonnant la Standard Edition, d'abord! En refusant les traductions qui aplatissent Freud, à tous les tournants : Ubersetzen - placer dessous! Comment faire autrement? Le message de Freud peut-il vivre dans une autre langue? Comment traduire Freud autrement qu'en devenant germaniste? Faut-il donc apprendre l'allemand quand on veut devenir analyste? Peut-être, mais cela ne suffit pas! Notre «retour à Freud», avec Lacan, exige le passage par la langue allemande, mais à condition de réaliser un "virage du sens à la lettre». Ne vous précipitez pas pour comprendre, en plaquant du sens, étudiez d'abord le jeu de la lettre, avant de prétendre que Freud avait voulu dire ceci ou cela. Si le «retour à Freud» n'exigeait que le passage par la langue allemande, les analystes allemands n'auraient nul besoin d'un «retour à Freud»; mais pour eux, souvent, la lecture de Freud est bien superficielle: "Vous croyez vraiment qu'il faut lire Freud?» Freud c'est le passé, dépassé! On ne lit plus que les auteurs américains ou anglais «à la pointe de la recherche»!

9. J. Lacan (1966): «D'un dessein », Écrits.

10. J. Lacan, Écrits, p. 365. 
Comment remettre en vigueur ce qui n'a cessé de soutenir la psychanalyse, dans sa déviation même, à savoir le sens premier que Freud préservait par sa seule présence? Cette découverte du pouvoir de la vérité, en nous et jusque dans notre chair, ne nous donne aucun pouvoir sur une vérité qui serait possédée par des sages, qui n'auraient plus qu'à la faire travailler à leur profit. Le savoir, que suppose l'acte analytique, se situe à la place de la vérité, mais peut-il se transmettre dans une institution? Ce savoir, élaboré par Freud, à partir de ses propres rêves, vaut-il pour tous? Un institut ne peut le transmettre sans que le sujet ne disparaisse dans la marge que Freud réserve à la vérité. Il n'y a pas de clef des songes permettant d'interpréter mécaniquement les rêves. Pas de savoir sans souffrance disaient les Grecs : Pathein - Mathein. Pas de transmission de la psychanalyse sans que l'effet de vérité ne s'opère au prix d'une perte. Et pas de traduction sans que le traducteur ne s'y brise. «L'impuissance à soutenir authentiquement une praxis, se rabat, comme il est, en l'histoire des hommes, commun, sur l'exercice d'un pouvoir». Et le traducteur s'empresse d'interpréter les passages litigieux, en tranchant dans le vif, au lieu de reconnaître les concepts freudiens qui sont à l'œuvre «dans leur ordonnance flexible mais impossible à rompre sans les dénouer». Puissamment articulés entre eux, ils «ne correspondent à rien qui se donne immédiatement à l'intuition». Tout «retour à Freud qui donne matière à un enseignement digne de ce nom, ne se produira que par la voie, par où la vérité la plus cachée se manifeste dans les révolutions de la culture. Cette voie est la seule formation que nous puissions prétendre transmettre à ceux qui nous suivent. Elle s'appelle un style». Y a-t-il un style pour traduire Freud, sans le trahir? La question est importante : nous précipiterons-nous sur le sens ou sur la lettre? "Qu'une de vos oreilles s'assourdisse, autant que l'autre doit être aiguë. Et c'est celle que vous devez tendre à l'écoute des sons ou phonèmes, des mots, des locutions, des sentances, sans y omettre pauses, scansions, coupes, périodes et parallélismes, car c'est là que se prépare le mot à mot de la version, faute de quoi l'intuition analytique est sans support et sans objet (Écrits, p. 471).

Peut-on traduire Freud, en respectant les jeux de la lettre, la métonymie, sans nous précipiter immédiatement sur la métaphore et le sens. Il en est de la traduction comme de l'institution: elle doit respecter le cheminement métonymique, elle ne doit pas le recouvrir d'une fluctuation métaphorique, hégémonique, qui s'arroge le pouvoir de dire la Vérité. C'est toute la différence entre le discours analytique et le discours du maître. Si l'un place le signifiant maitre " $S{ }^{1}$ ) en dominance, $\frac{S^{1}}{S}$ sur le sujet $(\boldsymbol{S})$, l'autre le place comme produit par le sujet $(\boldsymbol{S})$ (mais à condition que ce soit comme objet «a» que l'analyste se situe en place de semblant). Dès qu'il y a pouvoir, fut-ce de décider qui a raison ou tort, en ce qui concerne le sens qu'il convient d'attribuer à tel passage du texte freudien, nous sommes dans le registre de l'unaire, de l'univoque, qui impose la lecture «autorisée», authentique, «fidèle».

Il y a dans ce désir de fidélité un souci qui honore le traducteur, mais comment peut-il affirmer lui-même que sa traduction est «fidèle»? Tout traducteur s'affirme toujours «au plus près» du texte pour épouser la moindre nuance et respecter le rythme de la phrase autant que le sens du message. 
Nous savons qu'il ne suffit pas d'affirmer que l'épouse est fidèle : l'époux est souvent le dernier à être averti de l'aventure. C'est même sur ce détail qu'est construite «l'histoire des quarante femmes infidèles " ${ }^{11}$ : le grand Sultan, consterné de voir dans sa capitale tant de femmes infidèles, proclama un édit invitant les maris trompés à tuer leurs femmes, à condition qu'ils fussent sûrs de leur infortune. La proclamation n'indiquait ni le nombre, ni les noms de ces femmes, elle stipulait seulement que leur cas était connu de toute la ville: quarante femmes trompaient donc leurs maris, mais, comme toujours, les maris ignoraient tout de l'inconduite des épouses.

On s'attendait à un massacre dans les jours qui suivaient la proclamation, mais un mois entier s'écoula sans résultat, et le vizir proposait déjà au Sultan de proclamer la liste des femmes infidèles. - Non, dit le Sultan. Attendons encore, mon peuple est intelligent. Il se passera bientôt quelque chose!

Quarante jours après la proclamation, au cours de la nuit, les quarante femmes furent tuées. - Je ne comprends pas, dit le vizir. Pourquoi ont-ils agi tous le même jour?

- Très simple, dit le Sultan. Ils ont pu résoudre ce problème par pure déduction logique. Supposons qu'il n'y ait qu'une seule infidèle: tout le monde le sait, sauf le mari. Il croyait en la vertu de sa femme, mais comme il ne connaît dans la ville aucun cas d'infidélité (s'il y en avait, il le saurait), il doit en conclure qu'il ne peut s'agir que de sa femme. Il doit donc la tuer, dès la première nuit.

Supposons que deux femmes seulement soient infidèles. Abdullah savait bien que la femme d'Hadjibaba le trompait, et Hadjibaba en savait autant de la femme d'Abdullah. Donc le jour de la proclamation, chacun de ces deux hommes se dit: «Ah - ah! il va tuer sa femme», mais le lendemain les deux femmes sont encore en vie. Abdullah et Hadjibaba doivent donc penser qu'ils ont eu tort de croire à la fidélité de leur épouse. C'est pourquoi, la deuxième nuit, deux poignards doivent conclure ce raisonnement.

Si la femme de Farouk est aussi infidèle que la femme d'Abdullah et la femme d'Hadjibada, Farouk qui ne connaît que deux femmes infidèles, voyant que ses amis Abdullah et Hadjibaba n'ont pas assassiné, la seconde nuit, les infidèles, doit en conclure que sa femme est infidèle, elle aussi. Trois poignards doivent donc entrer en jeu la troisième nuit.

Et ainsi de suite, par induction mathématique, par déduction logique, par récurrence. Dois-je conclure que la fidélité d'une traduction ne s'apprécie, comme celle d'une épouse, que par un raisonnement analogue? Me faut-il obligatoirement passer par la référence à l'autre, à sa critique. Si personne ne me critique, c'est que «c'est bien». L'absence de négation peut n'être que temporaire : le silence de l'ensemble des traducteurs n'est pas une preuve. Mais le plus souvent, c'est par le biais d'une critique argumentée, vigoureuse que j'apprends que, sur tel ou tel point, ma traduction est «fautive». Contresens, faux-sens! Le professeur, ou l'universitaire, qui sommeille dans chaque traducteur s'em-

11. Raconté par G. GAMOW et M. STERN (1961): Les jeux mathématiques, Dunod. 
presse de réagir du haut de son Savoir placé en dominance sur le signifiant mâ̂tre, placé en vérité!

Le lecteur ne s'étonnera donc pas de se voir proposer dix-sept traductions, différentes, d'un même texte, relativement court. Quand nous nous sommes lancés «à nos risques et périls» dans l'aventure et les transports (Übertragung) de cette Übersetzung, nous étions loin de nous douter des difficultés du parcours. Allant de surprise en surprise, ce texte nous a menés et malmenés, comme aucun texte ne l'avait fait jusqu'ici. Dans un groupe de travail de l'École freudienne, intitulé «à partir de la Verneinung», nous avons repris avec R. Lew l'étude de «la Verneinung dans la théorie freudienne»12 et avec J. Rozenberg "Kant avec Freud» ${ }^{13}$. Qu'il s'agisse de la négation dans la logique aristotélicienne, ou chez Brentano, qu'il s'agisse de la négation dans la grammaire française (dans Pichon et Damourette) ou dans les rêves de nos patients, et dans nos propres rêves, dans les séminaires de Lacan, dans ses formules quantiques de sexuation, chez Marguerite Duras, chez Dostoïevski, dans le fantasme: «On bat un enfant», dans la logique moderne, dans la mise à distance de l'affect, nous n'avons cessé, pendant ces six dernières années, de nous référer à ce texte essentiel, traduit dans de nombreuses langues. Pour comprendre ce que chaque langue occulte, à sa façon, la Verneinung est un exemple merveilleux, mais nous ne pouvons, dans le cadre de cet article, nous lancer dans une étude comparative qui nous mènerait trop loin.

Contentons-nous de noter que, pour l'instant, nous n'avons toujours pas traduit le titre. Nous en étions restés à cette opposition et cette première scission : Société parisienne de psychanalyse - Société française de psychanalyse. Peut-on dire qu'à travers ces scissions les germanistes (Lacan lisait Freud dans le texte original) et les non-germanistes s'affrontaient. Il est de fait qu'à l'Institut, on citait Freud à partir de la "Standard Edition " (c'est déjà tout un programme!) alors qu'avec Lacan, on ne se référait à Freud qu'à travers le texte allemand. Nous ne pouvons ici recenser tous les auteurs français qui se sont penchés sur la Verneinung, mais après Hyppolite, il nous faut citer E. Benveniste (1956) qui, dans le premier numéro de la revue la Psychanalyse fit paraître ses «Remarques sur la fonction du langage dans la découverte freudienne». La Verneinung y était commentée avec pertinence ${ }^{14}$.

Aux environs de 1960, circulait, parmi les membres de la Société française de psychanalyse, une traduction intitulée «la dénégation». Ni datée, ni signée, elle était donnée venant de Laplanche. Sans vérifier cette attribution nous l'avions étudiée, dans le Coq Héron 52; elle y figurait en troisième position.

P. Levy, à l'usage des analystes de la Société parisienne, fit circuler une traduction intitulée «la dénégation». Elle corrigeait sur de nombreux points la traduction d'Hoesli et figurait en quatrième position dans notre étude.

12. Publié dans la revue du Coq Héron, $\mathrm{n}^{\circ} 55,1976$, Verneinung $\mathrm{I}$.

13. Publié dans le Coq Héron, $n^{\circ} 60,1977$, Verneinung II.

14. Benveniste a repris cette étude, en 1966, dans ses Problèmes de linguistique générale, (Ch. VIII), Paris, Gallimard. 

$\left(n^{\circ} 5\right)^{15}$

En 1971, F. Lyotard, dans «Discours, Figure», proposa sa traduction

En 1972, Bernard This entreprit, avec P. Lavalle, une traduction ( $\left.\mathrm{n}^{\circ} 6\right)$ qui fut présentée dans un «Cartel Psychose» de l'E.F.P.

En 1973, Irène Roublef et Solange Faladé, à partir de la Standard Edition (XIX, 233) nous avaient donné une traduction $\left(\mathrm{n}^{\circ} 7\right)$ qui fut étudiée dans le «Cartel Psychose».

Disposant des diverses traductions parues et les comparant phrase par phrase, nous avons pu, en 1973, proposer aux participants d'un groupe de travail ( «À partir de la Verneinung») une «lecture en relief» où les divergences-différences pouvaient apparaitre, et faire l'objet de nombreux commentaires.

En 1974, Jean Michel Rey, dans «Parcours de Freud» fit paraître sa traduction, accompagnée d'un long commentaire : "De la Dénégation " ${ }^{16}$ (trad. $n^{\circ} 8$ ).

En 1974, à partir des commentaires de notre groupe de travail, Pierre Thèves réalisa une traduction $\left(n^{\circ}\right.$ ) qui fut de nouveau longuement commentée, argumentée.

En février 1975, nous avons fait paraitre dans le Coq Héron $\mathrm{n}^{\circ} 52$ une traduction This-Thèves $\left(n^{\circ} 10\right)$ intitulée «Étude comparée de quelques traductions disponibles». Cette traduction était datée, (1975), et présentait le texte allemand avec en face la traduction française proposée temporairement; c'est dire que ce travail, suivi de nombreux commentaires, ne se proposait pas comme définitif ${ }^{17}$. Ce qui l'était, par contre, c'est notre certitude qu'il convenait, pour toute traduction de Freud, d'opposer page par page le texte allemand au texte français.

Hélas, qu'avions-nous fait en attribuant à Laplanche une traduction qui n'était pas de lui ? «Je n'ai jamais fait de traduction de la Verneinung du temps de la Société française de psychanalyse. Le texte auquel tu te réfères $m$ 'est d'ailleurs absolument inconnu ". Critiquant notre traduction et nous expliquant les raisons de son désaccord, Jean Laplanche, dans une longue lettre, nous fit parvenir une traduction inédite, réalisée vers 1967, au moment où il écrivait avec J.B. Pontalis le «Vocabulaire de la Psychanalyse». Si nous avions consulté cet ouvrage, nous aurions pu constater que Laplanche et Pontalis proposaient, à cette époque, de traduire Verneinung par (Dé)négation : «En allemand, Verneinung désigne la négation au sens logique et grammatical du terme... mais aussi la dénégation au sens psychologique (refus d'une affirmation que $\mathrm{j}$ 'ai énoncée ou qu'on m'impute, par exemple: non, je n'ai pas dit cela, je n'ai pas pensé cela). Il est impossible au traducteur d'opter à chaque passage pour «négation » ou «dénégation», la solution que nous proposons est de transcrire Verneinung en (dé)négation».

15. F, Lyotard: op. cit., p. 131-134. Klincksieck, France.

16. J.M. Rey : $o p$. cit., p. 115-261. Ed. Galilée. Tractatus.

17. C'est cette traduction que nous donnons en Appendice au texte de $B$. This et $P$. Thèves, ici-même - (F. Peraldi). 
Si nous n'admettions pas ces facilités d'écriture qui permettent aux traducteurs de laisser dans le flou le lecteur, en refusant de trancher entre négation et dénégation, il n'en restait pas moins que nous nous étions mis dans une situation difficile en attribuant à Jean Laplanche un texte qui n'était pas de lui. C'est pourquoi nous lui avons aussitôt demandé de bien vouloir accepter nos excuses, effaçant son nom des exemplaires du Coq Héron encore disponibles. Nous n'avons pas voulu critiquer la traduction qu'il nous avait fait parvenir, amicalement. Il y proposait d'écrire (Dé-)négation systématiquement, mais ce tiret ne changeait rien à l'affaire. Nous n'avons pas fait paraître notre réponse à ses "remarques", mais il nous y avait invités : "Nous poursuivrons, je l'espère, cette discussion passionnante et qui est loin d'être épuisée. Crois, je te prie, à ma sincère amitié».

Nous pensions être «au bout de nos peines » mais nous n'avions pas encore mesuré l'imprudence d'une démarche qui nous avait amenés à publier intégralement, pour son cinquantenaire (1925-1975) le texte allemand et la traduction proposée. Nous pensions, naïvement, qu'il appartenait au domaine public. Françoise Laye, au nom des Presses universitaires de France, nous fit observer qu'il n'en était rien: légalement une œuvre n'est «domaine public» que cinquante ans après la mort de l'auteur, mais en ce qui concerne Freud, il faut y ajouter les années de guerre qui comptent double. Cette «bévue» pouvait être considérée comme «atteinte aux droits des héritiers et des éditeurs». Heureusement notre travail fut reconnu dénué d'intention mercantile et... merci Françoise... il n'y eut pas de suite juridique.

Nous reprîmes notre travail à partir des remarques de Wachsberger, Klapahouk et Laplanche. Roger Dorey qui avait étudié notre traduction nous invita à son groupe de travail, avec les étudiants de Nanterre, et nous rencontrâmes Paul Felder, germaniste qui s'occupait des œuvres de Thomas Mann à Zurich. Leur traduction intitulée «La Négation» nous parvint en 1978 ( $\left.\mathrm{n}^{\circ} 14\right)$. Au cours de l'année 79 nous avons travaillé ensemble. R. Dorey nous procura une traduction de Colette Chiland intitulée «La Négation» $\left(n^{\circ} 13\right)$. Nous avions l'intention de produire une traduction commune, mais le travail n'avançait que bien lentement. Nous passions une soirée pour traduire quelques lignes, et le lendemain matin... nous n'étions déjà plus d'accord : nos points de vue divergeaient légèrement. Il était clair que chacun d'entre nous, pour traduire, se servait d'une théorisation implicite qui «déportait » la traduction. L'année scolaire s'achevant, les universitaires, débordés de travail, ne pouvaient multiplier les séances de traduction; nous avons décidé de suspendre nos réunions: la traduction de «la bande des quatre» ne verrait jamais le jour! Fallait-il faire paraître le texte de Freud et nos deux traductions? Le Coq Héron nous demandait de nous hâter, mais la traduction de Spitteler (Imago) et de Ferenczi accaparait nos énergies. Judith Dupont nous signala que G.A. Goldschmitt, écrivain et traducteur de Peter Handke, ayant lu notre travail, avait été intéressé par nos commentaires. Le Comité de rédaction du Coq Héron l'invita en juillet 79. Roger Dorey, Paul Felder, Suzanne Hommel, Françoise Männel, Christiane Stoll, Judith Dupont purent se joindre à nous, et de nouveau la Verneinung fut au coeur de nos préoccupations. Mais pour ne pas nous enliser dans des discussions inter- 
minables, Goldschmitt nous proposa une méthode de travail originale: il prit notre traduction du Coq Héron $\left(\mathrm{n}^{\circ} 10\right)$ et la traduisit en allemand. Nous avions le texte de Freud sous les yeux, et nous pouvions noter les divergences. Cette Rück-übersetzung s'avérait une excellente technique : nos erreurs apparaissaient aussitôt. Nous avons ainsi amélioré notre traduction, mais certains points restaient litigieux.

En 1979, Dorey et Felder ont fait paraître dans l'Évolution psychiatrique $\left(\mathrm{n}^{\circ} 72,1979\right.$, tome III) une nouvelle traduction $\left(\mathrm{n}^{\circ} 16\right)$ qui modifiait de nombreux détails (70) de leur travail précédent ( La négation»).

Nous avons repris leur travail en 1980 et élaboré cette dix-septième traduction, qui engage la communauté analytique française. Sans le travail de nos amis de l'Association psychanalytique de France, sans les remarques et critiques pertinentes de tous les germanistes qui ont pris contact avec nous, jamais nous n'aurions poursuivi notre travail de cette façon. Il est évident que cette $17 \mathrm{e}$ traduction améliore la première, mais elle n'est pas parfaite pour autant! Nous sommes particulièrement mécontents de n'avoir pu trouver une façon de faire apparaître l'idée de chute qui se trouve dans l'ein-fallen allemand. «La façon dont nos patients présentent ce qui leur vient à l'esprit pendant le travail analytique...». Dès la première ligne, les divergences apparaissent. La manière (1-3-4-5-5) dont nos patients exposent, proferent, avancent, expriment, énoncent (7-14) leurs associations (1-4-6) leurs idées (3-11), ce qui leur vient à l'esprit (3-4-5-7) pendant le travail analytique (3-4-5-7), au cours du traitement psychanalytique (1-6) nous fournit (1-6-7-11), nous donne lieu (3) à quelques observations (1-3-4-6-7-11) intéressantes.

Dans cette seule phrase nous voyons déjà que quelque chose se donne à lire qui n'est pas dans le texte de Freud. Einfalle pour ne prendre que ce mot, c'est ce qui «tombe dedans» (fallen-tomber), ce qui «s'écroule», ce qui fait irruption, incidence. Pouvions-nous traduire par «les associations»? Sûrement pas! Les associations, c'est Assoziationen. En parlant d'associations d'idées, nous modifions dangereusement la règle analytique qui prescrit à l'analysant d'exprimer (aus-drücken) tout ce qu'il imagine, pense, ressent, éprouve dans son corps. En mettant l'accent sur le «dire» (sagen) - «dire les idées», nous engageons l'analyse dans un processus de réflexion raisonnable où elle s'enlisera. Ce qui est à exprimer, c'est tout ce qui survient, fait irruption sans raison apparente. Nous avions pensé que le terme d' «incidente» utilisé grammaticalement pour désigner une proposition insérée dans une phrase pouvait traduire le mouvement de chute (cadere - tomber) exprimé dans Einfall. L' «incidente" survient inopinément, quand on ne l'attend pas; c'est comme le lapsus ou «l'acte manqué », une réussite du «dire». Il faudrait dire «ce qui leur tombe dedans», ou créer un mot: ce qui leur "en-tombe», comme on dit «enfonce». "Ce qui leur tombe dedans» (ou dessus, $\mathrm{n}^{\circ} 15$ ) est trop familier. Tout ce qui «tombe dedans" pose d'ailleurs le problème du lieu où ça tombe. Dans la tête ou dans le corps? Les traducteurs, innocemment, ont pensé qu'il s'agissait de la tête... ce qui tombe dans la tête ne peut être que la pensée. «Dites toutes vos pensées, toutes vos idées", et voilà l'analyse qui s'oriente sur la pente de l'intellectualisation, et voilà comment le corps et ses manifestations expressives pas- 
sent au second plan. Le signifiant, ce qui «fait signe»? Si la «psucho-ana-lysis» c'est ce qui «libère», délivre le souffle (c'est-à-dire le psuchos), pouvons-nous oublier que tout ce qui permet à l'homme de s'exprimer doit être accueilli dans l'analyse. En conservant, faute de mieux, temporairement, «ce qui leur vient à l'esprit», nous avons accepté de tomber dans le piège (Falle) de la langue française, mais nous en acceptons difficilement les limites! Il conviendra à partir des traductions anglaises, espagnoles, italiennes, japonaises... de montrer comment chaque langue efface tel ou tel aspect du texte freudien. Ce "défaut de traduction", que Freud appelait "refoulement ", nous l'avons nous aussi mis en acte de diverses façons. D'autres reprendrons ce travail, en d'autres langues, établissant cette circulation de sens que nous n'avons pu qu'amorcer. Nous avons «isolé» notre langue «maternelle» ${ }^{18}$ en privilégiant les traductions françaises. L'isolement est une «suppression des possibilités de contact, c'est un moyen de soustraire une chose à toute espèce de toucher, et quand le névrosé isole ainsi une impression ou une activité, par une pause, il nous donne symboliquement à comprendre qu'il ne veut pas laisser venir les pensées qui s'y rapportent en contact associatif avec d'autres" (Inhibition, symptôme et angoisse - G.W. XIV, p. 152). En rapprochant le français et l'allemand, les isolant des autres langues, nous avons établi un contact privilégié, mais nous en avons évité d'autres. Le toucher est «le but» de «l'investissement agressif" comme de «l'investissement tendre», notait Freud. Traduire, rapprocher des textes, est, dans cette perspective, une activité ambiguë, c'est tenter de «retrouver l'objet». Le plaisir primordial à retrouver le même (Wiederfinden das Gleichen in das Unheimliche) anime le traducteur: il rêve d'un texte qui ne serait que reflet, double, reproduction parfaite, dans une autre langue peut-être, mais sans contre-sens ni faux-sens, en respectant la pensée et le style. Le plaisir du traducteur c'est d'être précis, concis, fidèle, nous l'avons vu. Quelle jubilation quand le mot «juste» apparaît, et quand la périphrase s'élimine. Oh, l'étrange plaisir de la «Métaphasis»!

Entre le Witz et la traduction n'y aurait-il pas quelque rapprochement à établir? Le jeu d'esprit recherche la plus faible dépense d'énergie, et un refoulement moindre. La traduction cherche de la même façon cette prime de plaisir qui accompagne toute levée partielle du refoulement. Traduire-trahir, certes en participant à un refoulement incessant et inévitable: le travail du traducteur n'est pas neutre, il nous engage, nous l'avons vu, comme défenseur du texte de Freud, au côté des «fidèles», des fils respectueux, des frères compétitifs. "Voyez comme ils sont «fautifs", éventrant, renversant, écrasant tout sur leur passage». Ah, comme la «belle âme» peut se réjouir de ce travail, où les mots apparaissent travaillés par l'incessant glissement du sens, l'interminable procès de sémantisation. Cette réjouissance oublierait volontiers tout ce qu'elle doit au travail des autres. Nous avons, dans nos apostilles, marqué nos désaccords plus facilement que nos «heureuses surprises », devant les «trouvailles» de ceux qui faisaient progresser notre compréhension de ce texte, au cours de ce demisiècle de traduction. Remercions ici tous ceux qui nous ont permis de réaliser ce travail, sans oublier ceux qui, le reprenant, sauront le prolonger, l'améliorer!

18. Pourquoi est-elle plus « maternelle» que «paternelle»? Le père n'est pas un étranger! 
On pourrait nous dire que ce travail de traduction, qui vient éviter la Versagung der Übersetzung en «serrant» le texte «au plus près" pour conjurer le refoulement des signifiants et réduire l'écart, le détour, l'ajournement du sens, ne peut que s'inscrire dans une économie de transfert dominée par un vieux rêve de symétrie. Le désir de «ne faire qu'un» avec le texte, en épousant les plus légères inflexions de la phrase, en abolissant les distances et les barrières de la langue, c'est toujours cette même "Quête de l'Objet». Mettre à la place une réplique, un simulacre, une doublure, un calque, une copie... mais l'objet «retrouvé » ne sera jamais «le même» puisque l'être du sujet est perdu. Il y aura toujours une différence, et la traduction sera toujours "brèche» ne cessant de se creuser, compromis à renouveler, synthèse transitoire, il n'y a pas de traduction définitive.

On s'étonnera peut-être du plaisir manifesté par le traducteur quand il a réussi (du moins le croit-il!) à rendre la vie de la phrase et son style! Et son déplaisir, ses insomnies, son réveil au petit matin quand il peine et ne parvient pas à faire danser le texte, à mimer le mouvement de la phrase (ce qu'Aristote aurait appelé lexis - expression), tout en respectant la manifestation du sens (le Logos). Mais comment garder, dans la traduction, la trace de la récurrence des signifiants qui structurent le texte traduit? Quand nous brisons les réseaux de signifiants qui pourraient faire sens, le jeu des phonèmes qui jalonnent l'audition de la phrase (einfallen - Falle), pour satisfaire notre souci de précision et de clarté, en utilisant des termes (comme «incidente» et «piège») qui cessent de consonner, nous avons l'impression d'être comme l'éléphant dans le magasin de porcelaine. «Animal mimétique» par excellence, l'homme n'abandonne pas facilement ce matériel phonique, même s'il sait que la traduction est une transposition métaphorique substitutive (un signifiant $S$ remplacé par un autre S'). Si la métaphore tient tant de place dans la théorie aristotélicienne ${ }^{19}$ de l'activité poétique, c'est parce que, fondée sur la perception du semblable, elle constitue, plus qu'une figure parmi d'autres, le paradigme de toute activité de fiction.

Dans leur traduction de la Poétique d'Aristotélés, Roselyne Dupont-Roc et Jean Lallot ont choisi de traduire mimesis par «représentation» et non par «imitation», puisque le mot «mime» s'enracine dans une forme de représentation, au sens théâtral du terme (ce qui leur permet de maintenir «l'ambivalence de base de l'accusatif d'objet» - «représenter un objet», le «présentifier», mais le modèle ne peut se confondre avec sa copie. «Représenter» un homme n'est pas «imiter» un homme. La représentation implique une perte, une Katharsis, une épuration de l'émotion. Elle n'en permet pas moins l'apparition d'effets de surprise, dans ces renversements et changements (péripétie - coup de théâtre) imposés par la translation et la translittération. L'activité de traduction - métaphasie - est un art de passage, de métabase, de métabole; soumise à la contrainte du vraisemblable et de la nécessité, elle n'en est pas moins une activité poétique, dans la mesure où elle crée, à partir d'ob-

19. Curieusement, en français, Aristotélés (le meilleur, telos - but, fin) est devenu Aristote privé de la fin signifiante de son nom. 
jets préexistants, pour aboutir à une représentation qui procure le double plaisir d'apprendre $(\mu \alpha \nu \Theta \alpha \nu \Sigma, \nu)$ et de s'étonner $(\Theta \alpha v \mu \alpha \xi \Sigma \iota \nu)$ : un regard nouveau, une intelligence nouvelle.

Le traducteur, dit Blanchot ${ }^{20}$

est un écrivain d'une singulière originalité, précisément là où il paraît n'en revendiquer aucune. Il est le maître secret de la différence des langues, non pas pour l'abolir, mais pour l'utiliser, afin d'éveiller dans la sienne, par les changements violents ou subtils qu'il lui apporte, une présence de ce qu'il y a de différence originalement, dans l'original. Pas question, ici, de ressemblance, dit avec raison Benjamin; si l'on veut que l'œuvre traduite ressemble à l'œuvre à traduire, il n'y a pas de traduction littéraire possible. Il s'agit bien davantage d'une identité à partir d'une altérité, la même ceuvre dans deux langues étrangères et en raison de leur étrangeté et en rendant, par là, visible ce qui fait que cette ouvre sera toujours autre, mouvement dont il faut précisément tirer la lumière qui éclairera, par transparence, la traduction.

En traduisant, nous franchissons une frontière, nous transportons, déplaçons, différons, assurant un passage pour acquérir un point de vue nouveau. Übertragen - übersetzen - porter, placer au dessus. En allant d'une langue à l'autre, le traducteur sait bien qu'il n'y a pas d'équivalence terme à terme, les noyaux de sens étant parfois irréductibles. Il faut risquer, trancher, abandonner un mot pour un autre, préférer une formulation nouvelle, les mots résistant à ce déplacement, à ce report, à cette transcription: la castration est à l'xuvre, dans ce travail de réduction des écarts (élagage, allègement, aération) comme dans tout événement de discours. Mais dans l'écrit la castration s'incorpore tout particulièrement.

Dans son article sur «la sexualité féminine», Freud ${ }^{21}$ nous montre que la petite fille devait abandonner son premier objet, la mère, pour se tourner vers le père. Ce transfert (Übertragung) sur un substitut approprié, cette translation (Überführung), cette suscription (Überschreibung) écriture en surcharge, au dessus des liens affectifs unissant l'enfant à sa mère, c'est ce qui forme vraiment (bildet ja) le contenu principal du développement en femme (der zum Weibtum führenden Entwicklung).

On comprend que ce passage, de la mère au père, soit lié à la fonction de la métaphore du Nom du Père, métaphore qui se réalise dans cette substitution d'un signifiant ( $S$ - Désir de la Mère) par un autre signifiant $\left(\mathrm{S}^{1}\right.$ - nom du Père). À l'origine, l'enfant désire être le phallus, l'objet du désir de sa mère. Le père, inter-dicteur, en tant que marqué par l'interdit de l'inceste, sépare l'enfant de sa mère; il se dit «entre » eux et dans la mesure où il est «introduit» par la mère, il amène l'enfant à renoncer à la toute-puissance de son désir, acceptant la Loi qui est limitation, assomption du manque. Par son accès au Nom-du-père, l'enfant nomme son désir (le phallus) mais c'est au prix de l'aliéner: objet de son désir, le phallus est repoussé dans l'inconscient. C'est le refoulement originaire, qui détermine l'accès au langage. L'interdit sépare;

20. Blanchot: De l'amitié, p. 71-72.

21. S.F.: GW, p. 523. 
c'est le symbole de la négation, le «Non» (ne unum) qui permet l'indépendance de la pensée, du principe de plaisir. Si la négation permet l'activité de la pensée comme indépendante du principe de plaisir, elle permet aussi le

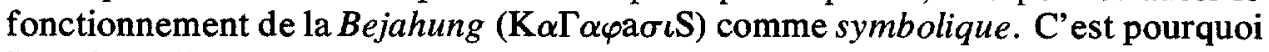
Freud souligne l'importance de la création du symbole de la négation: die Schöpfung des Verneinungssymbol. Les traducteurs (3-7-11) qui parlent du «symbole de la dénégation» rendent ce passage incompréhensible. Mais ce n'est pas parce que Freud, digne élève de F. Brentano, souligne l'importance de ce symbole, de cet opérateur logique, qu'il faut se croire obligé de traduire le titre de cet article par «la négation». Il faut en poursuivre la lecture jusqu'au bout, ce que nous n'avons pas fait ici, pour se rendre compte que l'essentiel de ce qu'apporte Freud, ce qu'il y a d'original dans son travail, c'est cette étude de la dénégation. «Ma mère, ce $n$ 'est pas elle». Nous rectifions : «donc, c'est sa mère»... Vous allez penser... mais ce n'est pas ma mère». Désexualisons. Dans les exemples évoqués par Freud, le sujet attribue son discours à l'autre: il peut s'approcher au plus près de son désir inconscient à condition de ne pas s'y reconnaître, mais d'y reconnaître un autre. La négation permet l'activité de la pensée, mais elle constitue la parole comme désexualisante : le sexe, ce qui pour être parlé doit d'abord être dénié.

Pour fonctionner, la pensée doit être désexualisée, mais, ce faisant, elle révèle sa fonction mortifere, la structuration intellectuelle «étant faite pour traduire sous forme de méconnaissance ce que la première symbolisation doit à la mort» (Lacan).

Avec la Verneinung l'inconscient (Unbewusste) n'est plus caractérisé négativement, en référence à la conscience. Dans une nouvelle économie du sujet, Freud cesse de le marquer seulement par des indices négatifs (ne connaît pas le temps, ne connaît pas la contradiction), c'est le $\mathrm{ICH}$, grand ou petit moi, qui fait usage de la négation, qui se présente «toujours en méconnaissance», travaillé, à son insu, par le langage. Ce langage, qu'il croit élaborer se présente comme un travail de reprise incessante, toujours en "déception d'énoncé" (J.M. REY). Le sujet ne peut s'écouter qu'en «après-coup» (nach-tragen) qu'à contretemps de son discours, dans le détour de l'événement voué irrémédiablement à la perte ${ }^{22}$.

Est-ce la mort qui nous apporte la question qui nie le discours? Freud, dans la Verneinung, ne pose pas explicitement le problème, il ne nous dit pas que c'est elle qui introduit la négation, mais il nous permet de comprendre que «la négativité du discours, en tant qu'elle fait être ce qui n'est pas, nous renvoie à la question de savoir ce que le Non-être, qui se manifeste dans l'ordre symbolique doit à la réalité de la mort». (Lacan)

Nous sommes là, à l'articulation du réel et du symbolique, de la mort et de la parole, et c'est notre symptôme. La mort engendre «le meurtre de la chose", il faut que la chose se perde pour être représentée par un préposé (Vor-stellen) qui masque la chose et en même temps la désigne.

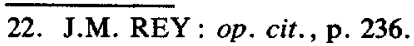


Avec les Wortvorstellungen, et l'introduction de la «pulsion de mort», la bascule de la seconde topique tournera autour de cette négativité essentielle de la parole qui s'imprime dans l'homme et le fait «parlêtre», être de parole. Les symboles enveloppent la vie de l'homme d'un réseau si total qu'ils conjoignent, avant qu'il vienne au monde, ceux qui vont l'engendrer.

Pour comprendre l'importance de la création symbolique de la négation (pas, qui, en français, vient «fendre» et animer le corps érigé, immobile) le texte freudien est essentiel. L'événement de la Verneinung, son «pas», qui est un franchissement, nous permettra peut-être de mieux comprendre pourquoi la répétition (Wiederholung) qui se présente comme l'insistance de la mort "détournée», ne peut que faire fleurir des traductions toujours différentes, toujours imprévisibles, dans l'impossibilité d'une Überschreibung définitive.

Le sujet sera toujours barré (\$), divisé : sujet de l'énoncé, sujet de l'énonciation; qu'il soit comme sujet toujours "jeté dessous», comme l'objet l'est «devant», c'est un fait de langue. Le traducteur sera toujours surpris par le système signifiant, et les effets de sa propre langue, et il ne cessera de s'en apercevoir: tradere c'est transmettre, faire passer d'une langue à l'autre - la tradition. Mais tradere c'est presque tradire - trahir.

La Tradition, transmission de l'héritage chrétien, est toujours menacée de trahison, quand le souffle de l'Esprit cesse de l'animer. Les Pères de l'Église (auctores) avaient pour mission d'augmenter (augere) la fécondité de la parole divine par leurs commentaires, et ils s'exprimaient, au Moyen Âge, en latin, langue écrite, véhicule du savoir, instrument du pouvoir de la Clergie ${ }^{23}$. Dante "pour offrir au peuple déshérité les miettes tombées de la table des anges» n'écrira pas le Banquet en latin, mais il précisera bien que le "savoir des doctes théologiens ne peut être reçu dans le «parler des illettrés»: «Et, à ce propos, que chacun sache que nulle chose, harmonisée par lien musaïque, ne peut se transmuer de sa parlure en une autre, sans rompre toute sa douceur et son harmonie». Il ne se fait pas d'illusions... et cependant il écrit.

Et nous? Nous traduisons le texte freudien, et nous le commentons, comme des Pères de l'Église? Comme des doctes, des maîtres, avec autorité? Avec d'incessants commentaires, pour marquer nos hésitations, bien sûr, mais aussi pour faire participer le lecteur au plaisir de nos découvertes. Sans dogmatisme, dans une glose incessante, novatrice, dans un constant travail de déconstruction-reconstruction du texte, et ceci au sein d'une École, institution qui, loin de détenir la Vérité, invite tout un chacun, selon son style, à s'exercer dans ce travail de commentaire. Cela suppose qu'un «lexique des concepts freudiens» soit créé, qui ne soit pas simple «vocabulaire de la psychanalyse». Le «retour à Freud» ne peut se contenter d'une lecture superficielle et d'une traduction «à la sauvette», par une seule personne, ou même une seule équipe ${ }^{24}$. Les concepts freudiens «reconnus dans leur ordonnance flexible mais impos-

23. Roger Dragonetti : «Auctor-autor-actor», dans Lettres de l'École, n 25, vol, I, p. 186 à 196.

24. C'est pourquoi la traduction française des cuvres de Freud, préparée par Pontalis, fait problème. Elle n'envisage même pas de nous donner le texte allemand et en face la traduction proposée, ce qui serait bien le minimum exigible. 
sible à rompre, sans les dénouer» décapés, lubrifiés, replacés là où ils manquaient, éventuellement, deviendront, traduits correctement, des rouages qui pourront fonctionner dans l'horlogerie freudienne.

S'en servir serait, opération nouvelle, découvrir qu'ils fonctionnent dans une structure rigoureusement construite. "Comment peut-on traduire Hafiz... ou Freud?" La formulation du titre de cet article laissait entendre: impossible!

Relire Freud, dans l'École de la Cause, c'est, avec le discours analytique mathématisé par Lacan, respecter le jeu et la vie de la lettre ${ }^{25}$ et faire apparaître les termes et concepts négligés, abandonnés, incompris, dans une première lecture trop rapide. Ce n'est pas imposer la fiction de sa traduction comme identique à la vérité (prendre la métaphore pour la métonymie), c'est reconnaître la vérité comme impossible à dire sans la fiction (reconnaître la métonymie dans la métaphore). Tout texte a des effets, en effet, en est fait, en naît fait, mais l'effet d'un texte peut-il réordonner les effets à venir? La traduction doitelle imposer un sens, interpréter le texte pour produire l'effet qui va de soi qui s'impose - refoulant tout autre effet? Ce serait confondre l'imaginaire et le réel. Nous ne pouvons pas nous passer de l'imaginaire, mais le «discours analytique » qui «imaginarise le réel du symbolique», dans la mesure où c'est le petit «a» qui s'adresse au sujet $(\boldsymbol{8})$ pour qu'il produise le signifiant $\left(\mathrm{S}^{1}\right)$, ne doit pas imposer l'Univoque à la place de l'équivoque, pour masquer la division des sujets. Qu'il puisse sortir des libertés, de la clôture d'une expérience, c'est ce qui tient à la nature de l'après-coup dans la signifiance ${ }^{26}$. Mais le traducteur ne peut se dire le serviteur de la vérité, sans construire un système qui l'exclut. Polyphème, devrait-il toujours signer son travail sous ce nom? «Beau nom pour l'inconscient» disait Lacan ${ }^{27}$. Beau nom pour un traducteur qui n'ignore pas les effets de l'après-coup. Nach-Trag, en allemand c'est l'après-coup mais c'est aussi le greffon, ce petit rien qui permet au rosier sauvage de porter, épanouie, parfumée, lumineuse, la rose, fruit de la culture, du cultellus, ce couteau, instrument de coupure.

25. Référence à R. Dragonetti (1980): La vie de la lettre au Moyen Âge, Paris, Seuil.

26. Lacan, Proposition du 9 octobre, p. 27.

27. Écrits, p. 667 . 